\title{
Some results on zero points of $m$-accretive operators in reflexive Banach spaces
}

\author{
Chang Qun Wu' ${ }^{1}$, Songtao $\mathrm{Lv}^{2^{*}}$ and Yunpeng Zhang ${ }^{3}$
}

\author{
"Correspondence: sqlvst@yeah.net \\ ${ }^{2}$ School of Mathematics and \\ Information Science, Shangqiu \\ Normal University, Shangqiu, \\ Henan, China \\ Full list of author information is \\ available at the end of the article
}

\begin{abstract}
A modified proximal point algorithm is proposed for treating common zero points of a finite family of $m$-accretive operators. A strong convergence theorem is established in a reflexive, strictly convex Banach space with the uniformly Gâteaux differentiable norm.
\end{abstract}

Keywords: accretive operator; nonexpansive mapping; resolvent; fixed point; zero point

\section{Introduction and preliminaries}

Let $E$ be a Banach space and let $E^{*}$ be the dual of $E$. Let $\langle\cdot, \cdot\rangle$ denote the pairing between $E$ and $E^{*}$. The normalized duality mapping $J: E \rightarrow 2^{E^{*}}$ is defined by

$$
J(x)=\left\{f \in E^{*}:\langle x, f\rangle=\|x\|^{2}=\|f\|^{2}\right\}, \quad \forall x \in E .
$$

A Banach space $E$ is said to strictly convex if and only if $\|x\|=\|y\|=\|(1-\lambda) x+\lambda y\|$ for $x, y \in E$ and $0<\lambda<1$ implies that $x=y$. Let $U_{E}=\{x \in E:\|x\|=1\}$. The norm of $E$ is said to be Gâteaux differentiable if the limit $\lim _{t \rightarrow 0} \frac{\|x+t y\|-\|x\|}{t}$ exists for each $x, y \in U_{E}$. In this case, $E$ is said to be smooth. The norm of $E$ is said to be uniformly Gâteaux differentiable if for each $y \in U_{E}$, the limit is attained uniformly for all $x \in U_{E}$. The norm of $E$ is said to be Fréchet differentiable if for each $x \in U_{E}$, the limit is attained uniformly for all $y \in U_{E}$. The norm of $E$ is said to be uniformly Fréchet differentiable if the limit is attained uniformly for all $x, y \in U_{E}$. It is well known that (uniform) Fréchet differentiability of the norm of $E$ implies (uniform) Gâteaux differentiability of the norm of $E$.

Let $\rho_{E}:[0, \infty) \rightarrow[0, \infty)$ be the modulus of smoothness of $E$ by

$$
\rho_{E}(t)=\sup \left\{\frac{\|x+y\|-\|x-y\|}{2}-1: x \in U_{E},\|y\| \leq t\right\} .
$$

A Banach space $E$ is said to be uniformly smooth if $\frac{\rho_{E}(t)}{t} \rightarrow 0$ as $t \rightarrow 0$. It is well known that if the norm of $E$ is uniformly Gâteaux differentiable, then the duality mapping $J$ is single valued and uniformly norm to weak* continuous on each bounded subset of $E$.

Recall that a closed convex subset $C$ of a Banach space $E$ is said to have a normal structure if for each bounded closed convex subset $K$ of $C$ which contains at least two points, there exists an element $x$ of $K$ which is not a diametral point of $K$, i.e., $\sup \{\|x-y\|: y \in$ $K\}<d(K)$, where $d(K)$ is the diameter of $K$.

○2014 Wu et al.; licensee Springer. This is an Open Access article distributed under the terms of the Creative Commons Attribution License (http://creativecommons.org/licenses/by/2.0), which permits unrestricted use, distribution, and reproduction in any medium, provided the original work is properly cited. 
Let $D$ be a nonempty subset of a set $C$. Let $\operatorname{Proj}_{D}: C \rightarrow D$. $Q$ is said to be

(1) sunny if for each $x \in C$ and $t \in(0,1)$, we have $\operatorname{Proj}_{D}\left(t x+(1-t) \operatorname{Proj}_{D} x\right)=\operatorname{Proj}_{D} x$;

(2) a contraction if $\operatorname{Proj}_{D}^{2}=\operatorname{Proj}_{D}$;

(3) a sunny nonexpansive retraction if $\operatorname{Proj}_{D}$ is sunny, nonexpansive, and a contraction.

$D$ is said to be a nonexpansive retract of $C$ if there exists a nonexpansive retraction from $C$ onto $D$. The following result, which was established in [1-3], describes a characterization of sunny nonexpansive retractions on a smooth Banach space.

Let $E$ be a smooth Banach space and let $C$ be a nonempty subset of $E$. Let $\operatorname{Proj}_{C}: E \rightarrow C$ be a retraction and $J_{\varphi}$ be the duality mapping on $E$. Then the following are equivalent:

(1) $\operatorname{Proj}_{C}$ is sunny and nonexpansive;

(2) $\left\langle x-\operatorname{Proj}_{C} x, J_{\varphi}\left(y-\operatorname{Proj}_{C} x\right)\right\rangle \leq 0, \forall x \in E, y \in C$;

(3) $\left\|\operatorname{Proj}_{C} x-\operatorname{Proj}_{C} y\right\|^{2} \leq\left\langle x-y, J_{\varphi}\left(\operatorname{Proj}_{C} x-\operatorname{Proj}_{C} y\right)\right\rangle, \forall x, y \in E$.

It is well known that if $E$ is a Hilbert space, then a sunny nonexpansive retraction $\operatorname{Proj}_{C}$ is coincident with the metric projection from $E$ onto $C$. Let $C$ be a nonempty closed convex subset of a smooth Banach space $E$, let $x \in E$, and let $x_{0} \in C$. Then we have from the above that $x_{0}=\operatorname{Proj}_{C} x$ if and only if $\left\langle x-x_{0}, J_{\varphi}\left(y-x_{0}\right)\right\rangle \leq 0$ for all $y \in C$, where $\operatorname{Proj}_{C}$ is a sunny nonexpansive retraction from $E$ onto $C$. For more additional information on nonexpansive retracts, see [4] and the references therein.

Let $C$ be a nonempty closed convex subset of $E$. Let $T: C \rightarrow C$ be a mapping. In this paper, we use $F(T)$ to denote the set of fixed points of $T$. Recall that $T$ is said to be an $\alpha$-contractive mapping iff there exists a constant $\alpha \in[0,1)$ such that $\|T x-T y\| \leq \alpha \| x-$ $y \|, \forall x, y \in C$. The Picard iterative process is an efficient method to study fixed points of $\alpha$-contractive mappings. It is well known that $\alpha$-contractive mappings have a unique fixed point. $T$ is said to be nonexpansive iff $\|T x-T y\| \leq\|x-y\|, \forall x, y \in C$. It is well known that nonexpansive mappings have fixed points if the set $C$ is closed and convex, and the space $E$ is uniformly convex. The Krasnoselski-Mann iterative process is an efficient method for studying fixed points of nonexpansive mappings. The Krasnoselski-Mann iterative process generates a sequence $\left\{x_{n}\right\}$ in the following manner:

$$
x_{1} \in C, \quad x_{n+1}=\alpha_{n} T x_{n}+\left(1-\alpha_{n}\right) x_{n}, \quad \forall n \geq 1 .
$$

It is well known that the Krasnoselski-Mann iterative process only has weak convergence for nonexpansive mappings in infinite-dimensional Hilbert spaces; see [5-7] for more details and the references therein. In many disciplines, including economics, image recovery, quantum physics, and control theory, problems arise in infinite-dimensional spaces. In such problems, strong convergence (norm convergence) is often much more desirable than weak convergence, for it translates the physically tangible property that the energy $\left\|x_{n}-x\right\|$ of the error between the iterate $x_{n}$ and the solution $x$ eventually becomes arbitrarily small. To improve the weak convergence of a Krasnoselski-Mann iterative process, so-called hybrid projections have been considered; see [8-22] for more details and the references therein. The Halpern iterative process was initially introduced in [23]; see [23] for more details and the references therein. The Halpern iterative process generates a sequence $\left\{x_{n}\right\}$ in the following manner:

$$
x_{1} \in C, \quad x_{n+1}=\alpha_{n} u+\left(1-\alpha_{n}\right) T x_{n}, \quad \forall n \geq 1,
$$


where $x_{1}$ is an initial and $u$ is a fixed element in $C$. Strong convergence of Halpern iterative process does not depend on metric projections. The Halpern iterative process has recently been extensively studied for treating accretive operators; see [24-31] and the references therein.

Let $I$ denote the identity operator on $E$. An operator $A \subset E \times E$ with domain $D(A)=\{z \in$ $E: A z \neq \emptyset\}$ and range $R(A)=\bigcup\{A z: z \in D(A)\}$ is said to be accretive if for each $x_{i} \in D(A)$ and $y_{i} \in A x_{i}, i=1,2$, there exists $j\left(x_{1}-x_{2}\right) \in J\left(x_{1}-x_{2}\right)$ such that $\left\langle y_{1}-y_{2}, j\left(x_{1}-x_{2}\right)\right\rangle \geq 0$. An accretive operator $A$ is said to be $m$-accretive if $R(I+r A)=E$ for all $r>0$. In this paper, we use $A^{-1}(0)$ to denote the set of zero points of $A$. For an accretive operator $A$, we can define a nonexpansive single valued mapping $J_{r}: R(I+r A) \rightarrow D(A)$ by $J_{r}=(I+r A)^{-1}$ for each $r>0$, which is called the resolvent of $A$.

Now, we are in a position to give the lemmas to prove main results.

Lemma 1.1 [32] Let $\left\{a_{n}\right\},\left\{b_{n}\right\},\left\{c_{n}\right\}$, and $\left\{d_{n}\right\}$ be four nonnegative real sequences satisfying $a_{n+1} \leq\left(1-b_{n}\right) a_{n}+b_{n} c_{n}+d_{n}, \forall n \geq n_{0}$, where $n_{0}$ is some positive integer, $\left\{b_{n}\right\}$ is a number sequence in $(0,1)$ such that $\sum_{n=n_{0}}^{\infty} b_{n}=\infty,\left\{c_{n}\right\}$ is a number sequence such that $\limsup _{n \rightarrow \infty} c_{n} \leq 0$, and $\left\{d_{n}\right\}$ is a positive number sequence such that $\sum_{n=n_{0}}^{\infty} d_{n}<\infty$. Then $\lim _{n \rightarrow \infty} a_{n}=0$.

Lemma 1.2 [33] Let $C$ be a closed convex subset of a strictly convex Banach space E. Let $N \geq 1$ be some positive integer and let $T_{i}: C \rightarrow C$ be a nonexpansive mapping for each $i \in\{1,2, \ldots, N\}$. Let $\left\{\delta_{i}\right\}$ be a real number sequence in $(0,1)$ with $\sum_{i=1}^{N} \delta_{i}=1$. Suppose that $\bigcap_{i=1}^{N} F\left(T_{i}\right)$ is nonempty. Then the mapping $\bigcap_{i=1}^{N} T_{i}$ is defined to be nonexpansive with $F\left(\bigcap_{i=1}^{N} T_{i}\right)=\bigcap_{i=1}^{N} F\left(T_{i}\right)$.

Lemma 1.3 [34] Let $\left\{x_{n}\right\}$ and $\left\{y_{n}\right\}$ be bounded sequences in a Banach space $E$ and let $\beta_{n}$ be a sequence in $[0,1]$ with $0<\liminf _{n \rightarrow \infty} \beta_{n} \leq \limsup _{n \rightarrow \infty} \beta_{n}<1$. Suppose that $x_{n+1}=$ $\left(1-\beta_{n}\right) y_{n}+\beta_{n} x_{n}$ for all $n \geq 0$ and

$$
\limsup _{n \rightarrow \infty}\left(\left\|y_{n+1}-y_{n}\right\|-\left\|x_{n+1}-x_{n}\right\|\right) \leq 0 .
$$

Then $\lim _{n \rightarrow \infty}\left\|y_{n}-x_{n}\right\|=0$.

Lemma 1.4 [35] Let E be a real reflexive Banach space with the uniformly Gâteaux differentiable norm and the normal structure, and let $C$ be a nonempty closed convex subset of $E$. Let $f: C \rightarrow C$ be $\alpha$-contractive mapping and let $T: C \rightarrow C$ be a nonexpansive mapping with a fixed point. Let $\left\{x_{t}\right\}$ be a sequence generated by the following: $x_{t}=t f\left(x_{t}\right)+(1-t) T x_{t}$, where $t \in(0,1)$. Then $\left\{x_{t}\right\}$ converges strongly as $t \rightarrow 0$ to a fixed point $x^{*}$ of $T$, which is the unique solution in $F(T)$ to the following variational inequality: $\left\langle f\left(x^{*}\right)-x^{*}, j\left(x^{*}-p\right)\right\rangle \geq 0$, $\forall p \in F(T)$.

\section{Main results}

Theorem 2.1 Let E be a real reflexive, strictly convex Banach space with the uniformly Gâteaux differentiable norm. Let $N \geq 1$ be some positive integer. Let $A_{m}$ be an m-accretive operator in $E$ for each $m \in\{1,2, \ldots, N\}$. Assume that $C:=\bigcap_{m=1}^{N} \overline{D\left(A_{m}\right)}$ is convex and has the normal structure. Let $f: C \rightarrow C$ be an $\alpha$-contractive mapping. Let $\left\{\alpha_{n}\right\},\left\{\beta_{n}\right\}$, and $\left\{\gamma_{n}\right\}$ be real number sequences in $(0,1)$ with the restriction $\alpha_{n}+\beta_{n}+\gamma_{n}=1$. Let $\left\{\delta_{n, i}\right\}$ be a real number sequence in $(0,1)$ with the restriction $\delta_{n, 1}+\delta_{n, 2}+\cdots+\delta_{n, N}=1$. Let $\left\{r_{m}\right\}$ be a positive 
real numbers sequence and $\left\{e_{n, i}\right\}$ a sequence in $E$ for each $i \in\{1,2, \ldots, N\}$. Assume that $\bigcap_{i=1}^{N} A_{i}^{-1}(0)$ is not empty. Let $\left\{x_{n}\right\}$ be a sequence generated in the following manner:

$$
x_{1} \in C, \quad x_{n+1}=\alpha_{n} f\left(x_{n}\right)+\beta_{n} x_{n}+\gamma_{n} \sum_{i=1}^{N} \delta_{n, i} J_{r_{i}}\left(x_{n}+e_{n, i}\right), \quad \forall n \geq 1,
$$

where $J_{r_{i}}=\left(I+r_{i} A_{i}\right)^{-1}$. Assume that the control sequences $\left\{\alpha_{n}\right\},\left\{\beta_{n}\right\},\left\{\gamma_{n}\right\}$, and $\left\{\delta_{n, i}\right\}$ satisfy the following restrictions:

(a) $\lim _{n \rightarrow \infty} \alpha_{n}=0, \sum_{n=1}^{\infty} \alpha_{n}=\infty$;

(b) $0<\liminf _{n \rightarrow \infty} \beta_{n} \leq \lim \sup _{n \rightarrow \infty} \beta_{n}<1$;

(c) $\sum_{n=1}^{\infty}\left\|e_{n, m}\right\|<\infty$;

(d) $\lim _{n \rightarrow \infty} \delta_{n, i}=\delta_{i} \in(0,1)$.

Then the sequence $\left\{x_{n}\right\}$ converges strongly to $\bar{x}$, which is the unique solution to the following variational inequality: $\langle f(\bar{x})-\bar{x}, J(p-\bar{x})\rangle \leq 0, \forall p \in \bigcap_{i=1}^{N} A_{i}^{-1}(0)$.

Proof Put $y_{n}=\sum_{i=1}^{N} \delta_{n, i} J_{r_{i}}\left(x_{n}+e_{n, i}\right)$. Fixing $p \in \bigcap_{i=1}^{N} A_{i}^{-1}(0)$, we have

$$
\begin{aligned}
\left\|y_{n}-p\right\| & \leq \sum_{i=1}^{N} \delta_{n, i}\left\|J_{r_{i}}\left(x_{n}+e_{n, i}\right)-p\right\| \\
& \leq \sum_{i=1}^{N} \delta_{n, i}\left\|\left(x_{n}+e_{n, i}\right)-p\right\| \\
& \leq\left\|x_{n}-p\right\|+\sum_{i=1}^{N}\left\|e_{n, i}\right\| .
\end{aligned}
$$

Hence, we have

$$
\begin{aligned}
\left\|x_{n+1}-p\right\| \leq & \alpha_{n}\left\|f\left(x_{n}\right)-p\right\|+\beta_{n}\left\|x_{n}-p\right\|+\gamma_{n}\left\|y_{n}-p\right\| \\
\leq & \alpha_{n} \alpha\left\|x_{n}-p\right\|+\alpha_{n}\|f(p)-p\|+\beta_{n}\left\|x_{n}-p\right\|+\gamma_{n}\left\|x_{n}-p\right\|+\gamma_{n} \sum_{i=1}^{N}\left\|e_{n, i}\right\| \\
\leq & \left(1-\alpha_{n}(1-\alpha)\right)\left\|x_{n}-p\right\|+\alpha_{n}(1-\alpha) \frac{\|f(p)-p\|}{1-\alpha}+\sum_{i=1}^{N}\left\|e_{n, i}\right\| \\
\leq & \max \left\{\left\|x_{n}-p\right\|,\|f(p)-p\|\right\}+\sum_{i=1}^{N}\left\|e_{n, i}\right\| \\
& \vdots \\
\leq & \max \left\{\left\|x_{1}-p\right\|,\|f(p)-p\|\right\}+\sum_{j=1}^{\infty} \sum_{i=1}^{N}\left\|e_{j, i}\right\| .
\end{aligned}
$$

This proves that the sequence $\left\{x_{n}\right\}$ is bounded, and so is $\left\{y_{n}\right\}$. Since

$$
\begin{aligned}
y_{n}-y_{n-1}= & \sum_{i=1}^{N} \delta_{n, i}\left(J_{r_{m}}\left(x_{n}+e_{n, i}\right)-J_{r_{i}}\left(x_{n-1}+e_{n-1, i}\right)\right) \\
& +\sum_{i=1}^{N}\left(\delta_{n, i}-\delta_{n-1, i}\right) J_{r_{i}}\left(x_{n-1}+e_{n-1, i}\right),
\end{aligned}
$$


we have

$$
\begin{aligned}
\left\|y_{n}-y_{n-1}\right\| \leq & \sum_{i=1}^{N} \delta_{n, i}\left\|J_{r_{i}}\left(x_{n}+e_{n, i}\right)-J_{r_{i}}\left(x_{n-1}+e_{n-1, i}\right)\right\| \\
& +\sum_{i=1}^{N}\left|\delta_{n, i}-\delta_{n-1, i}\right|\left\|J_{r_{i}}\left(x_{n-1}+e_{n-1, i}\right)\right\| \\
\leq & \left\|x_{n}-x_{n-1}\right\|+\sum_{i=1}^{N}\left\|e_{n, i}\right\|+\sum_{i=1}^{N}\left\|e_{n-1, i}\right\| \\
& +\sum_{i=1}^{N}\left|\delta_{n, i}-\delta_{n-1, i}\right|\left\|J_{r_{i}}\left(x_{n-1}+e_{n-1, i}\right)\right\| \\
\leq & \left\|x_{n}-x_{n-1}\right\|+\sum_{i=1}^{N}\left\|e_{n, i}\right\|+\sum_{i=1}^{N}\left\|e_{n-1, i}\right\|+M_{1} \sum_{i=1}^{N}\left|\delta_{n, i}-\delta_{n-1, i}\right|
\end{aligned}
$$

where $M_{1}$ is an appropriate constant such that

$$
M_{1}=\max \left\{\sup _{n \geq 1}\left\|J_{r_{1}}\left(x_{n}+e_{n, 1}\right)\right\|, \sup _{n \geq 1}\left\|J_{r_{2}}\left(x_{n}+e_{n, 2}\right)\right\|, \ldots, \sup _{n \geq 1}\left\|J_{r_{N}}\left(x_{n}+e_{n, N}\right)\right\|\right\} .
$$

Define a sequence $\left\{z_{n}\right\}$ by $z_{n}:=\frac{x_{n+1}-\beta_{n} x_{n}}{1-\beta_{n}}$, that is, $x_{n+1}=\beta_{n} x_{n}+\left(1-\beta_{n}\right) z_{n}$. It follows that

$$
\begin{aligned}
\left\|y z_{n}-z_{n-1}\right\| \leq & \frac{\alpha_{n}}{1-\beta_{n}}\left\|f\left(x_{n}\right)-y_{n}\right\|+\frac{\alpha_{n-1}}{1-\beta_{n-1}}\left\|f\left(x_{n-1}\right)-y_{n-1}\right\|+\left\|y_{n}-y_{n-1}\right\| \\
\leq & \frac{\alpha_{n}}{1-\beta_{n}}\left\|f\left(x_{n}\right)-y_{n}\right\|+\frac{\alpha_{n-1}}{1-\beta_{n-1}}\left\|f\left(x_{n-1}\right)-y_{n-1}\right\|+\left\|x_{n}-x_{n-1}\right\| \\
& +\sum_{i=1}^{N}\left|\delta_{n, i}-\delta_{n-1, i}\right|\left\|J_{r_{i}} x_{n-1}\right\| \\
\leq & \frac{\alpha_{n}}{1-\beta_{n}}\left\|f\left(x_{n}\right)-y_{n}\right\|+\frac{\alpha_{n-1}}{1-\beta_{n-1}}\left\|f\left(x_{n-1}\right)-y_{n-1}\right\|+\left\|x_{n}-x_{n-1}\right\| \\
& +M_{2}\left(\sum_{i=1}^{N}\left|\delta_{n, i}-\delta_{i}\right|+\sum_{i=1}^{N}\left|\delta_{i}-\delta_{n-1, i}\right|\right)
\end{aligned}
$$

where $M_{2}$ is an appropriate constant such that

$$
M_{2}=\max \left\{\sup _{n \geq 1}\left\|J_{r_{1}} x_{n}\right\|, \sup _{n \geq 1}\left\|J_{r_{2}} x_{n}\right\|, \ldots, \sup _{n \geq 1}\left\|J_{r_{N}} x_{n}\right\|\right\} .
$$

This implies that

$$
\begin{aligned}
& \left\|z_{n}-z_{n-1}\right\|-\left\|x_{n}-x_{n-1}\right\| \\
& \leq \frac{\alpha_{n}}{1-\beta_{n}}\left\|f\left(x_{n}\right)-y_{n}\right\|+\frac{\alpha_{n-1}}{1-\beta_{n-1}}\left\|f\left(x_{n-1}\right)-y_{n-1}\right\| \\
& \quad+M_{2}\left(\sum_{i=1}^{N}\left|\delta_{n, i}-\delta_{i}\right|+\sum_{i=1}^{N}\left|\delta_{i}-\delta_{n-1, i}\right|\right) .
\end{aligned}
$$


From the restrictions (a), (b), (c), and (d), we find that

$$
\limsup _{n \rightarrow \infty}\left(\left\|z_{n}-z_{n-1}\right\|-\left\|x_{n}-x_{n-1}\right\|\right) \leq 0
$$

Using Lemma 1.4, we find that $\lim _{n \rightarrow \infty}\left\|z_{n}-x_{n}\right\|=0$. This further shows that $\lim \sup _{n \rightarrow \infty}\left\|x_{n+1}-x_{n}\right\|=0$. Put $T=\sum_{i=1}^{N} \delta_{i} J_{r_{i}}$. It follows from Lemma 1.3 that $T$ is nonexpansive with $F(T)=\bigcap_{i=1}^{N} F\left(J_{r_{i}}\right)=\bigcap_{i=1}^{N} A_{i}^{-1}(0)$. Note that

$$
\begin{aligned}
& \left\|x_{n}-T x_{n}\right\| \\
& \quad \leq\left\|x_{n}-x_{n+1}\right\|+\left\|x_{n+1}-T x_{n}\right\| \\
& \quad \leq\left\|x_{n}-x_{n+1}\right\|+\alpha_{n}\left\|f\left(x_{n}\right)-T x_{n}\right\|+\beta_{n}\left\|x_{n}-T x_{n}\right\|+\gamma_{n}\left\|y_{n}-T x_{n}\right\| \\
& \quad \leq\left\|x_{n}-x_{n+1}\right\|+\alpha_{n}\left\|f\left(x_{n}\right)-T x_{n}\right\|+\beta_{n}\left\|x_{n}-T x_{n}\right\|+M_{2} \sum_{i=1}^{N}\left|\delta_{n, i}-\delta_{i}\right| .
\end{aligned}
$$

This implies that

$$
\left(1-\beta_{n}\right)\left\|x_{n}-T x_{n}\right\| \leq\left\|x_{n}-x_{n+1}\right\|+\alpha_{n}\left\|f\left(x_{n}\right)-T x_{n}\right\|+M_{2} \sum_{i=1}^{N}\left|\delta_{n, i}-\delta_{i}\right| .
$$

It follows from the restrictions (a), (b), and (d) that

$$
\lim _{n \rightarrow \infty}\left\|T x_{n}-x_{n}\right\|=0 .
$$

Now, we are in a position to prove that $\limsup _{n \rightarrow \infty}\left\langle f(\bar{x})-\bar{x}, J\left(x_{n}-\bar{x}\right)\right\rangle \leq 0$, where $\bar{x}=$ $\lim _{t \rightarrow 0} x_{t}$, and $x_{t}$ solves the fixed point equation

$$
x_{t}=t f\left(x_{t}\right)+(1-t) T x_{t}, \quad \forall t \in(0,1) .
$$

It follows that

$$
\begin{aligned}
\left\|x_{t}-x_{n}\right\|^{2}= & t\left\langle f\left(x_{t}\right)-x_{n}, J\left(x_{t}-x_{n}\right)\right\rangle+(1-t)\left\langle T x_{t}-x_{n}, j\left(x_{t}-x_{n}\right)\right\rangle \\
= & t\left\langle f\left(x_{t}\right)-x_{t}, J\left(x_{t}-x_{n}\right)\right\rangle+t\left(x_{t}-x_{n}, J\left(x_{t}-x_{n}\right)\right\rangle \\
& +(1-t)\left\langle T x_{t}-T x_{n}, J\left(x_{t}-x_{n}\right)\right\rangle+(1-t)\left\langle T x_{n}-x_{n}, J\left(x_{t}-x_{n}\right)\right\rangle \\
\leq & t\left\langle f\left(x_{t}\right)-x_{t}, J\left(x_{t}-x_{n}\right)\right\rangle+\left\|x_{t}-x_{n}\right\|^{2}+\left\|T x_{n}-x_{n}\right\|\left\|x_{t}-x_{n}\right\|, \quad \forall t \in(0,1) .
\end{aligned}
$$

This implies that

$$
\left\langle x_{t}-f\left(x_{t}\right), J\left(x_{t}-x_{n}\right)\right\rangle \leq \frac{1}{t}\left\|T x_{n}-x_{n}\right\|\left\|x_{t}-x_{n}\right\|, \quad \forall t \in(0,1) .
$$

Since $\lim _{n \rightarrow \infty}\left\|T x_{n}-x_{n}\right\|=0$, we find that $\limsup _{n \rightarrow \infty}\left\langle x_{t}-f\left(x_{t}\right), J\left(x_{t}-x_{n}\right)\right\rangle \leq 0$. Since $J$ is strong to weak* uniformly continuous on bounded subsets of $E$, we find that

$$
\begin{aligned}
& \left|\left\langle f(\bar{x})-\bar{x}, J\left(x_{n}-\bar{x}\right)\right\rangle-\left\langle x_{t}-f\left(x_{t}\right), J\left(x_{t}-x_{n}\right)\right\rangle\right| \\
& \quad \leq\left|\left\langle f(\bar{x})-\bar{x}, J\left(x_{n}-\bar{x}\right)\right\rangle-\left\langle f(\bar{x})-\bar{x}, J\left(x_{n}-x_{t}\right)\right\rangle\right|
\end{aligned}
$$




$$
\begin{aligned}
& +\left|\left\langle f(\bar{x})-\bar{x}, J\left(x_{n}-x_{t}\right)\right\rangle-\left\langle x_{t}-f\left(x_{t}\right), J\left(x_{t}-x_{n}\right)\right\rangle\right| \\
\leq & \left|\left\langle f(\bar{x})-\bar{x}, J\left(x_{n}-\bar{x}\right)-J\left(x_{n}-x_{t}\right)\right\rangle\right|+\left|\left\langle f(\bar{x})-\bar{x}+x_{t}-f\left(x_{t}\right), J\left(x_{n}-x_{t}\right)\right\rangle\right| \\
\leq & \left\|f\left(x_{t}\right)-\bar{x}\right\|\left\|J\left(x_{n}-\bar{x}\right)-J\left(x_{n}-x_{t}\right)\right\|+(1+\alpha)\left\|\bar{x}-x_{t}\right\|\left\|x_{n}-x_{t}\right\| .
\end{aligned}
$$

Since $x_{t} \rightarrow \bar{x}$, as $t \rightarrow 0$, we have

$$
\lim _{t \rightarrow 0}\left|\left\langle f(\bar{x})-\bar{x}, J\left(x_{n}-\bar{x}\right)\right\rangle-\left\langle f\left(x_{t}\right)-x_{t}, J\left(x_{n}-x_{t}\right)\right\rangle\right|=0 .
$$

For $\epsilon>0$, there exists $\delta>0$ such that $\forall t \in(0, \delta)$, we have

$$
\left\langle f(\bar{x})-\bar{x}, J\left(x_{n}-\bar{x}\right)\right\rangle \leq\left\langle f\left(x_{t}\right)-x_{t}, J\left(x_{n}-x_{t}\right)\right\rangle+\epsilon .
$$

This implies that $\limsup _{n \rightarrow \infty}\left\langle f(\bar{x})-\bar{x}, J\left(x_{n}-\bar{x}\right)\right\rangle \leq 0$.

Finally, we show that $x_{n} \rightarrow \bar{x}$ as $n \rightarrow \infty$. Since $\|\cdot\|^{2}$ is convex, we see that

$$
\begin{aligned}
\left\|y_{n}-\bar{x}\right\|^{2} & =\left\|\sum_{i=1}^{N} \delta_{n, i} J_{r_{i}}\left(x_{n}+e_{n, i}\right)-\bar{x}\right\|^{2} \\
& \leq \sum_{i=1}^{N} \delta_{n, i}\left\|J_{r_{i}}\left(x_{n}+e_{n, i}\right)-\bar{x}\right\|^{2} \\
& \leq\left\|x_{n}-\bar{x}\right\|^{2}+\sum_{i=1}^{N}\left\|e_{n, i}\right\|\left(\left\|e_{n, i}\right\|+2\left\|x_{n}-\bar{x}\right\|\right) .
\end{aligned}
$$

It follows that

$$
\begin{aligned}
\left\|x_{n+1}-\bar{x}\right\|^{2}= & \alpha_{n}\left\langle f\left(x_{n}\right)-\bar{x}, J\left(x_{n+1}-\bar{x}\right)\right\rangle+\beta_{n}\left\langle x_{n}-\bar{x}, J\left(x_{n+1}-\bar{x}\right)\right\rangle \\
& +\gamma_{n}\left\langle y_{n}-\bar{x}, J\left(x_{n+1}-\bar{x}\right)\right\rangle \\
\leq & \alpha_{n} \alpha\left\|x_{n}-\bar{x}\right\|\left\|x_{n+1}-\bar{x}\right\|+\alpha_{n}\left\langle f(\bar{x})-\bar{x}, J\left(x_{n+1}-\bar{x}\right)\right\rangle \\
& +\beta_{n}\left\|x_{n}-\bar{x}\right\|\left\|x_{n+1}-\bar{x}\right\|+\gamma_{n}\left\|y_{n}-\bar{x}\right\|\left\|x_{n+1}-\bar{x}\right\| \\
\leq & \frac{\alpha_{n} \alpha}{2}\left(\left\|x_{n}-\bar{x}\right\|^{2}+\left\|x_{n+1}-\bar{x}\right\|^{2}\right)+\alpha_{n}\left\langle f(\bar{x})-\bar{x}, J\left(x_{n+1}-\bar{x}\right)\right\rangle \\
& +\frac{\beta_{n}}{2}\left(\left\|x_{n}-\bar{x}\right\|^{2}+\left\|x_{n+1}-\bar{x}\right\|^{2}\right)+\frac{\gamma_{n}}{2}\left\|x_{n}-\bar{x}\right\|^{2} \\
& +\sum_{i=1}^{N}\left\|e_{n, i}\right\|\left(\left\|e_{n, i}\right\|+2\left\|x_{n}-\bar{x}\right\|\right)+\frac{\gamma_{n}}{2}\left\|x_{n+1}-\bar{x}\right\|^{2} .
\end{aligned}
$$

Hence, we have

$$
\begin{aligned}
\left\|x_{n+1}-\bar{x}\right\|^{2} \leq & \left(1-\alpha_{n}(1-\alpha)\right)\left\|x_{n}-\bar{x}\right\|^{2}+2 \alpha_{n}\left\langle f(\bar{x})-\bar{x}, J\left(x_{n+1}-\bar{x}\right)\right\rangle \\
& +\sum_{i=1}^{N}\left\|e_{n, i}\right\|\left(\left\|e_{n, i}\right\|+2\left\|x_{n}-\bar{x}\right\|\right) .
\end{aligned}
$$

Using Lemma 1.1, we find $x_{n} \rightarrow \bar{x}$ as $n \rightarrow \infty$. This completes the proof. 
Remark 2.2 There are many spaces satisfying the restriction in Theorem 2.1, for example $L^{p}$, where $p>1$.

Corollary 2.3 Let $E$ be a Hilbert space and let $N \geq 1$ be some positive integer. Let $A_{m}$ be a maximal monotone operator in $E$ for each $m \in\{1,2, \ldots, N\}$. Assume that $C:=\bigcap_{m=1}^{N} \overline{D\left(A_{m}\right)}$ is convex and has the normal structure. Let $f: C \rightarrow C$ be an $\alpha$-contractive mapping. Let $\left\{\alpha_{n}\right\},\left\{\beta_{n}\right\}$, and $\left\{\gamma_{n}\right\}$ be real number sequences in $(0,1)$ with the restriction $\alpha_{n}+\beta_{n}+\gamma_{n}=1$. Let $\left\{\delta_{n, i}\right\}$ be a real number sequence in $(0,1)$ with the restriction $\delta_{n, 1}+\delta_{n, 2}+\cdots+\delta_{n, N}=1$. Let $\left\{r_{m}\right\}$ be a positive real numbers sequence and $\left\{e_{n, i}\right\}$ a sequence in $E$ for each $i \in\{1,2, \ldots, N\}$. Assume that $\bigcap_{i=1}^{N} A_{i}^{-1}(0)$ is not empty. Let $\left\{x_{n}\right\}$ be a sequence generated in the following manner:

$$
x_{1} \in C, \quad x_{n+1}=\alpha_{n} f\left(x_{n}\right)+\beta_{n} x_{n}+\gamma_{n} \sum_{i=1}^{N} \delta_{n, j} J_{r_{i}}\left(x_{n}+e_{n, i}\right), \quad \forall n \geq 1,
$$

where $J_{r_{i}}=\left(I+r_{i} A_{i}\right)^{-1}$. Assume that the control sequences $\left\{\alpha_{n}\right\},\left\{\beta_{n}\right\},\left\{\gamma_{n}\right\}$, and $\left\{\delta_{n, i}\right\}$ satisfy the following restrictions:

(a) $\lim _{n \rightarrow \infty} \alpha_{n}=0, \sum_{n=1}^{\infty} \alpha_{n}=\infty$;

(b) $0<\liminf _{n \rightarrow \infty} \beta_{n} \leq \lim \sup _{n \rightarrow \infty} \beta_{n}<1$;

(c) $\sum_{n=1}^{\infty}\left\|e_{n, m}\right\|<\infty$;

(d) $\lim _{n \rightarrow \infty} \delta_{n, i}=\delta_{i} \in(0,1)$.

Then the sequence $\left\{x_{n}\right\}$ converges strongly to $\bar{x}$, which is the unique solution to the following variational inequality: $\langle f(\bar{x})-\bar{x}, p-\bar{x}\rangle \leq 0, \forall p \in \bigcap_{i=1}^{N} A_{i}^{-1}(0)$.

\section{Applications}

In this section, we consider a variational inequality problem. Let $A: C \rightarrow E^{*}$ be a single valued monotone operator which is hemicontinuous; that is, continuous along each line segment in $C$ with respect to the weak* topology of $E^{*}$. Consider the following variational inequality:

find $x \in C$ such that $\langle y-x, A x\rangle \geq 0, \quad \forall y \in C$.

The solution set of the variational inequality is denoted by $\operatorname{VI}(C, A)$. Recall that the normal cone $N_{C}(x)$ for $C$ at a point $x \in C$ is defined by

$$
N_{C}(x)=\left\{x^{*} \in E^{*}:\left\langle y-x, x^{*}\right\rangle \leq 0, \forall y \in C\right\} .
$$

Now, we are in a position to give the convergence theorem.

Theorem 3.1 Let E be a real reflexive, strictly convex Banach space with the uniformly Gâteaux differentiable norm. Let $N \geq 1$ be some positive integer and let $C$ be nonempty closed and convex subset of E. Let $A_{i}: C \rightarrow E^{*}$ a single valued, monotone and hemicontinuous operator. Assume that $\bigcap_{i=1}^{N} \mathrm{VI}\left(C, A_{i}\right)$ is not empty and $C$ has the normal structure. Let $f: C \rightarrow C$ be an $\alpha$-contractive mapping. Let $\left\{\alpha_{n}\right\},\left\{\beta_{n}\right\}$, and $\left\{\gamma_{n}\right\}$ be real number sequences in $(0,1)$ with the restriction $\alpha_{n}+\beta_{n}+\gamma_{n}=1$. Let $\left\{\delta_{n, i}\right\}$ be a real number sequence in $(0,1)$ with the restriction $\delta_{n, 1}+\delta_{n, 2}+\cdots+\delta_{n, N}=1$. Let $\left\{r_{m}\right\}$ be a positive real numbers sequence 
and $\left\{e_{n, i}\right\}$ a sequence in $E$ for each $i \in\{1,2, \ldots, N\}$. Let $\left\{x_{n}\right\}$ be a sequence generated in the following manner:

$$
x_{1} \in C, \quad x_{n+1}=\alpha_{n} f\left(x_{n}\right)+\beta_{n} x_{n}+\gamma_{n} \sum_{i=1}^{N} \delta_{n, i} \mathrm{VI}\left(C, A_{i}+\frac{1}{r_{i}}\left(I-x_{n}\right)\right), \quad \forall n \geq 1 .
$$

Assume that the control sequences $\left\{\alpha_{n}\right\},\left\{\beta_{n}\right\},\left\{\gamma_{n}\right\}$, and $\left\{\delta_{n, i}\right\}$ satisfy the following restrictions:

(a) $\lim _{n \rightarrow \infty} \alpha_{n}=0, \sum_{n=1}^{\infty} \alpha_{n}=\infty$;

(b) $0<\liminf _{n \rightarrow \infty} \beta_{n} \leq \lim \sup _{n \rightarrow \infty} \beta_{n}<1$;

(c) $\sum_{n=1}^{\infty}\left\|e_{n, m}\right\|<\infty$;

(d) $\lim _{n \rightarrow \infty} \delta_{n, i}=\delta_{i} \in(0,1)$.

Then the sequence $\left\{x_{n}\right\}$ converges strongly to $\bar{x}$, which is the unique solution to the following variational inequality: $\langle f(\bar{x})-\bar{x}, J(p-\bar{x})\rangle \leq 0, \forall p \in \bigcap_{i=1}^{N} \operatorname{VI}\left(C, A_{i}\right)$.

Proof Define a mapping $T_{i} \subset E \times E^{*}$ by

$$
T_{i} x:= \begin{cases}A_{i} x+N_{C} x, & x \in C, \\ \emptyset, & x \notin C .\end{cases}
$$

From Rockafellar [36], we find that $T_{i}$ is maximal monotone with $T_{i}^{-1}(0)=\operatorname{VI}\left(C, A_{i}\right)$. For each $r_{i}>0$, and $x_{n} \in E$, we see that there exists a unique $x_{r_{i}} \in D\left(T_{i}\right)$ such that $x_{n} \in x_{r_{i}}+$ $r_{i} T_{i}\left(x_{r_{i}}\right)$, where $x_{r_{i}}=\left(I+r_{i} T_{i}\right)^{-1} x_{n}$. Notice that

$$
y_{n, i}=\mathrm{VI}\left(C, A_{i}+\frac{1}{r_{i}}\left(I-x_{n}\right)\right)
$$

which is equivalent to

$$
\left\langle y-y_{n, i}, A_{i} y_{n, i}+\frac{1}{r_{i}}\left(y_{n, i}-x_{n}\right)\right\rangle \geq 0, \quad \forall y \in C,
$$

that is, $-A_{i} y_{n, i}+\frac{1}{r_{i}}\left(x_{n}-y_{n, i}\right) \in N_{C}\left(y_{n, i}\right)$. This implies that $y_{n, i}=\left(I+r_{i} T_{i}\right)^{-1} x_{n}$. Using Theorem 2.1, we find the desired conclusion immediately.

From Theorem 3.1, the following result is not hard to derive.

Corollary 3.2 Let E be a real reflexive, strictly convex Banach space with the uniformly Gâteaux differentiable norm. Let $C$ be nonempty closed and convex subset of E. Let $A: C \rightarrow$ $E^{*}$ a single valued, monotone and hemicontinuous operator with $\operatorname{VI}(C, A)$. Assume that $C$ has the normal structure. Let $f: C \rightarrow C$ be an $\alpha$-contractive mapping. Let $\left\{\alpha_{n}\right\},\left\{\beta_{n}\right\}$, and $\left\{\gamma_{n}\right\}$ be real number sequences in $(0,1)$ with the restriction $\alpha_{n}+\beta_{n}+\gamma_{n}=1$. Let $\left\{x_{n}\right\}$ be a sequence generated in the following manner:

$$
x_{1} \in C, \quad x_{n+1}=\alpha_{n} f\left(x_{n}\right)+\beta_{n} x_{n}+\gamma_{n} \operatorname{VI}\left(C, A+\frac{1}{r}\left(I-x_{n}\right)\right), \quad \forall n \geq 1 .
$$

Assume that the control sequences $\left\{\alpha_{n}\right\},\left\{\beta_{n}\right\}$, and $\left\{\gamma_{n}\right\}$ satisfy the following restrictions: 
(a) $\lim _{n \rightarrow \infty} \alpha_{n}=0, \sum_{n=1}^{\infty} \alpha_{n}=\infty$;

(b) $0<\liminf _{n \rightarrow \infty} \beta_{n} \leq \limsup _{n \rightarrow \infty} \beta_{n}<1$.

Then the sequence $\left\{x_{n}\right\}$ converges strongly to $\bar{x}$, which is the unique solution to the following variational inequality: $\langle f(\bar{x})-\bar{x}, J(p-\bar{x})\rangle \leq 0, \forall p \in \operatorname{VI}\left(C, A_{i}\right)$.

\section{Competing interests}

The authors declare that they have no competing interests.

Authors' contributions

All authors contributed equally to this manuscript. All authors read and approved the final manuscript.

\section{Author details}

${ }^{1}$ School of Business and Administration, Henan University, Kaifeng, Henan, China. ${ }^{2}$ School of Mathematics and Information Science, Shangqiu Normal University, Shangqiu, Henan, China. ${ }^{3}$ Vietnam National University, Hanoi, Vietnam.

\section{Acknowledgements}

The authors are grateful to the editor and the reviewers for useful suggestions which improved the contents of the article.

\section{Received: 16 January 2014 Accepted: 30 April 2014 Published: 14 May 2014}

\section{References}

1. Bruck, RE: Nonexpansive projections on subsets of Banach spaces. Pac. J. Math. 47, 341-355 (1973)

2. Reich, S: Asymptotic behavior of contractions in Banach spaces. J. Math. Anal. Appl. 44, 57-70 (1973)

3. Goebel, K, Reich, S: Uniform Convexity, Hyperbolic Geometry, and Nonexpansive Mappings. Dekker, New York (1984)

4. Kopecká, EE, Reich, S: Nonexpansive retracts in Banach spaces. Banach Cent. Publ. 77, 161-174 (2007)

5. Bauschke, HH, Matousková, E, Reich, S: Projection and proximal point methods: convergence results and counterexamples. Nonlinear Anal. 56, 715-738 (2004)

6. Reich, S: Weak convergence theorems for nonexpansive mappings in Banach spaces. J. Math. Anal. Appl. 67, 274-276 (1979)

7. Genel, A, Lindenstruss, J: An example concerning fixed points. Isr. J. Math. 22, 81-86 (1975)

8. Cho, SY, Li, W, Kang, SM: Convergence analysis of an iterative algorithm for monotone operators. J. Inequal. Appl. 2013, Article ID 199 (2013)

9. Cho, SY, Qin, X: On the strong convergence of an iterative process for asymptotically strict pseudocontractions and equilibrium problems. Appl. Math. Comput. 235, 430-438 (2014)

10. Yang, S, Zhang, MY: Strong convergence theorems for fixed points of generalized asymptotically quasi- $\phi$-nonexpansive mappings. Adv. Fixed Point Theory 4, 69-90 (2014)

11. Cho, SY, Qin, X, Kang, SM: Hybrid projection algorithms for treating common fixed points of a family of demicontinuous pseudocontractions. Appl. Math. Lett. 25, 854-857 (2012)

12. Zhou, $\mathrm{H}$ : Convergence theorems of common fixed points for a family of Lipschitz quasi-pseudocontractions. Nonlinear Anal. 71, 685-690 (2009)

13. Qin, X, Agarwal, RP: Shrinking projection methods for a pair of asymptotically quasi- $\phi$-nonexpansive mappings Numer. Funct. Anal. Optim. 31, 1072-1089 (2010)

14. Chen, JH: Iterations for equilibrium and fixed point problems. J. Nonlinear Funct. Anal. 2013, Article ID 4 (2013)

15. Hao, Y: Some results on a modified Mann iterative scheme in a reflexive Banach space. Fixed Point Theory Appl. 2013, Article ID 227 (2013)

16. Kim, JK: Strong convergence theorems by hybrid projection methods for equilibrium problems and fixed point problems of the asymptotically quasi- $\boldsymbol{\phi}$-nonexpansive mappings. Fixed Point Theory Appl. 2011, Article ID 10 (2011)

17. Qin, X, Cho, SY, Kang, SM: Strong convergence of shrinking projection methods for quasi- $\phi$-nonexpansive mappings and equilibrium problems. J. Comput. Appl. Math. 234, 750-760 (2010)

18. Wu, C, Sun, L: A monotone projection algorithm for fixed points of nonlinear operators. Fixed Point Theory Appl. 2013, Article ID 318 (2013)

19. Wu, C, Lv, S: Bregman projection methods for zeros of monotone operators. J. Fixed Point Theory 2013, Article ID 7 (2013)

20. Qin, X, Su, Y: Strong convergence theorems for relatively nonexpansive mappings in a Banach space. Nonlinear Anal. 67, 1958-1965 (2007)

21. Ye, J, Huang, J: Strong convergence theorems for fixed point problems and generalized equilibrium problems of three relatively quasi-nonexpansive mappings in Banach spaces. J. Math. Comput. Sci. 1, 1-18 (2011)

22. Qin, X, Cho, YJ, Kang, SM: Convergence theorems of common elements for equilibrium problems and fixed point problems in Banach spaces. J. Comput. Appl. Math. 225, 20-30 (2009)

23. Halpern, B: Fixed points of nonexpanding maps. Bull. Am. Math. Soc. 73, 957-961 (1967)

24. Qin, X, Cho, SY, Wang, L: A regularization method for treating zero points of the sum of two monotone operators. Fixed Point Theory Appl. 2014, Article ID 75 (2014)

25. Takahashi, W: Viscosity approximation methods for resolvents of accretive operators in Banach spaces. J. Fixed Point Theory Appl. 1, 135-147 (2007)

26. Song, J, Chen, M: A modified Mann iteration for zero points of accretive operators. Fixed Point Theory Appl. 2013, Article ID 347 (2013)

27. Qin, X, Su, Y: Approximation of a zero point of accretive operator in Banach spaces. J. Math. Anal. Appl. 329, 415-424 (2007) 
28. Yang, S: Zero theorems of accretive operators in reflexive Banach spaces. J. Nonlinear Funct. Anal. 2013, Article ID 2 (2013)

29. Qing, Y, Cho, SY: A regularization algorithm for zero points of accretive operators. Fixed Point Theory Appl. 2013 Article ID 341 (2013)

30. Qing, Y, Cho, SY: Proximal point algorithms for zero points of nonlinear operators. Fixed Point Theory Appl. 2014, Article ID 42 (2014)

31. Reich, S: Strong convergence theorems for resolvents of accretive operators in Banach spaces. J. Math. Anal. Appl. 75 287-292 (1980)

32. Liu, LS: Ishikawa and Mann iterative process with errors for nonlinear strongly accretive mappings in Banach spaces. J. Math. Anal. Appl. 194, 114-125 (1995)

33. Bruck, RE: Properties of fixed-point sets of nonexpansive mappings in Banach spaces. Trans. Am. Math. Soc. 179, 251-262 (1973)

34. Chang, SS: Some problems and results in the study of nonlinear analysis. Nonlinear Anal. 30, 4197-4208 (1997)

35. Qin, X, Cho, SY, Wang, L: Iterative algorithms with errors for zero points of $m$-accretive operators. Fixed Point Theory Appl. 2013, Article ID 148 (2013)

36. Rockafellar, RT: On the maximality of sums of nonlinear monotone operators. Trans. Am. Math. Soc. 149, 75-88 (1970)

10.1186/1687-1812-2014-118

Cite this article as: Wu et al:: Some results on zero points of $m$-accretive operators in reflexive Banach spaces. Fixed Point Theory and Applications 2014, 2014:118

\section{Submit your manuscript to a SpringerOpen ${ }^{\circ}$ journal and benefit from:}

- Convenient online submission

- Rigorous peer review

- Immediate publication on acceptance

- Open access: articles freely available online

- High visibility within the field

- Retaining the copyright to your article 